Research Article

\title{
Optimal Investment-Reinsurance Policy with Stochastic Interest and Inflation Rates
}

\author{
Xin Zhang (iD) ${ }^{1}$ and Xiaoxiao Zheng ${ }^{2}$ \\ ${ }^{1}$ School of Mathematics, Southeast University, Nanjing, Jiangsu 211189, China \\ ${ }^{2}$ Department of Fixed Income, Bohai Securities Co., Ltd., Tianjin 300381, China \\ Correspondence should be addressed to Xin Zhang; x.zhang.seu@gmail.com
}

Received 13 September 2019; Revised 13 November 2019; Accepted 2 December 2019; Published 17 December 2019

Academic Editor: Alberto Cavallo

Copyright (c) 2019 Xin Zhang and Xiaoxiao Zheng. This is an open access article distributed under the Creative Commons Attribution License, which permits unrestricted use, distribution, and reproduction in any medium, provided the original work is properly cited.

\begin{abstract}
The aim of this paper is to study a classic problem in actuarial mathematics, namely, an optimal reinsurance-investment problem, in the presence of stochastic interest and inflation rates. This is of relevance since insurers make investment and risk management decisions over a relatively long horizon where uncertainty about interest rate and inflation rate may have significant impacts on these decisions. We consider the situation where three investment opportunities, namely, a savings account, a share, and a bond, are available to an insurer in a security market. In the meantime, the insurer transfers part of its insurance risk through acquiring a proportional reinsurance. The investment and reinsurance decisions are made so as to maximize an expected power utility on terminal wealth. An explicit solution to the problem is derived for each of the two well-known stochastic interest rate models, namely, the Ho-Lee model and the Vasicek model, using standard techniques in stochastic optimal control theory. Numerical examples are presented to illustrate the impacts of the two different stochastic interest rate modeling assumptions on optimal decision making of the insurer.
\end{abstract}

\section{Introduction}

The purpose of this paper is to investigate a classic problem in actuarial mathematics, namely, an optimal reinsurance-investment problem, in the presence of stochastic interest and inflation rates. Investment and risk management decisions of insurance companies are, by their nature, long-term decision making. Over a relatively long period of time, there could be significant changes in interest rate and inflation rate, and these changes are usually difficult to be anticipated at the time while the decisions are made. Consequently, the incorporation of random movements of these rates in modeling decision making of insurance companies may be of practical interest and relevance. In this paper, two stochastic interest rate models, namely, the Ho-Lee model and the Vasicek model, are considered. To incorporate a realistic feature of inflation rate, namely, mean reversion, we adopt a meanreverting time-dependent Ornstein-Uhlenbeck (OU) process. A diffusion approximation is employed to model the surplus process of an insurance company. To incorporate the impact of inflation on the surplus process, which is relevant due to the long-term nature of the decision making problems of the insurance company, the surplus process is adjusted to the price index level reflecting inflation. We consider the situation where the insurance company can transfer its risk attributed to insurance liabilities to a reinsurance company by means of proportional reinsurance, which is one of the popular reinsurance treaties. The insurance company may also diversify its risk and make profits from investing in a security market. It is supposed here that there are three investment opportunities available to the insurance company in a security market, namely, a risky share, a zero-coupon bond, and a saving account. The aim of the insurance company is to determine a combined optimal investment and reinsurance policy with a view to maximizing the expected power utility on the company's terminal wealth. Using the HamiltonJacobi-Bellman (HJB) dynamic programming approach, an explicit solution to the optimal investment and reinsurance 
problem of the insurance company is obtained in each of the two stochastic interest rate models, namely, the Ho-Lee model and the Vasicek model. The verification theorems for the HJB solutions to the optimal investment and reinsurance problem for the two stochastic interest rate models are provided. Numerical results based on hypothetical parameter values are presented to illustrate the impacts of the two different stochastic interest rate modeling assumptions on optimal decision making of the insurer. In what follows, a brief review on some relevant literature and the motivation of this study are provided.

The optimal investment-reinsurance problem is one of the classic research topics in actuarial and insurance mathematics. It aims to provide a theoretical basis for rational decision making of insurers on investments in a security market and transferring insurance risks to reinsurance companies. The rational decisions of the insurers are made by optimizing certain objective criteria such as utility function. This is related to the utility maximization problem in neoclassical economics as well as financial economics. The solution of the optimization problem is, by its nature, normative, though results of positive nature may occasionally obtain. In financial economics, a "formal" study of an optimal investment problem may be tracked back to the pioneering work by Markowitz [1], where the scientific foundation of the problem was enacted by making use of mathematics. Specifically, the problem was formulated mathematically as an optimization problem in a static oneperiod model, where only the expected return of a portfolio and the risk of the portfolio measured by the variance of its return were relevant. More realistic situations, such as continuous-time situations, were considered in the second stage of developments pioneered by the seminal articles by Merton [2, 3]. In particular, the continuous-time optimal asset allocation model by Merton [2, 3] brought the historical developments of the field in a new era and stimulated the development of continuous-time finance (see, for example, Siu [4] for related discussion). Since the seminal works by Merton [2, 3], there have been many significant developments in continuous-time optimal asset allocation models and a large volume of literature on the field. It does not seem to be easy to list all of them here, but some of them are mentioned without slighting the other literature. One may refer to the monographs by Merton [5], Ralf [6], Karatzas and Shreve [7], Elliott and Kopp [8], and the relevant literature therein for more detailed exposition on the developments of the literature in the field. Davis and Norman [9] investigated an optimal consumption and investment decision for an investor who invests in a simplified security market with a bank account and a stock. Furthermore, the authors took fixed percentage transaction costs into consideration. A life-cycle model of consumption and portfolio was considered by Cocco et al. [10]. Kraft [11] studied an optimal portfolio selection problem with Heston's stochastic volatility.

Long-term strategic asset allocation is one of the important areas in optimal asset allocation. It sheds light on optimal investment decision making in financial planning, insurance, and pension funds. Intuitively, factors such as inflation, interest rate, investment cycle, and economic cycle which seem to be relevant to long-term investment decision making may be taken into account when discussing longterm strategic asset allocation. The monograph by Campbell and Viceira [12] provides an excellent account of long-term strategic asset allocation, where the problem was rigorously treated in discrete time modeling frameworks. There is also a considerable amount of finance and insurance literature on long-term strategic asset allocation. Again some literature studies, particularly those relating to incorporation of inflation and interest rate, are mentioned here. Inflation has a significant impact on long-term investment decision making. Indeed, inflation was used as the main driver of investment return series in long run in one of the major models in actuarial science, namely, the Wilkie stochastic investment model, developed by Wilkie [13]. A key feature of inflation, namely, mean reversion, was described by an autoregressive time series model (in discrete time) in the Wilkie stochastic investment model (see, for example, Siu [4] for related discussion). Pearson and Sun [14] described the inflation risk by a mean-reverting square-root process, while in Munk et al. [15] and Brennan and Xia [16], the inflation process is modeled by an Ornstein-Uhlenbeck process. Siu [4] discussed a long-term strategic asset allocation problem in continuous time by incorporating inflation and regime switching. Korn et al. [17] and Siu [18] considered an optimal asset allocation of pension funds in the presence of both the inflation and regime switching risks using a martingale approach and a backward stochastic differential equation approach, respectively. See also the relevant literature in the papers by Siu [4], Korn et al. [17], and Siu [18] for some works on optimal asset allocation in the presence of inflation. Apart from inflation risk, the interest risk is another important factor that we have to face. Korn and Kraft [19] studied the portfolio problems with stochastic interest rates. In $\mathrm{Li}$ and $\mathrm{Wu}[20]$, stochastic interest rate is given by the Cox-Ingersoll-Ross (CIR) model and the volatility of the stock is also a CIR process. Kraft [21] considered all common short rate models and stochastic discount. Shen and Siu [22] investigated an optimal asset allocation problem in the presence of both stochastic interest rate and regime switching effect, where a regime-switching version of the Vasicek model was used to describe stochastic interest rate.

There has been a large amount of work on optimal investment of an insurance company. Some of the literature studies are, for example, Wang et al. [23], Zeng [24], Luo et al. [25], Elliott and Siu [26], Liang et al. [27], Elliott and Siu [28], and Siu [29]. For an insurance company, it is natural to adopt a prudent investment strategy; that is, they should not only diversify risk by investing their money into different assets but also should avoid the risk brought from the company's relatively long investment cycle. Over a long period of time, accumulated inflation can lead to huge shrinkage of the wealth. What is more relevant here is a combined optimal investment and reinsurance problem of an insurer. Some of the literature is also mentioned here. Bai and Zhang [30] considered an optimal investment-reinsurance problem for an insurer under the mean-variance criterion. Zhang and Siu [31] incorporated model 
uncertainty, or ambiguity, in an optimal investment-reinsurance problem by adopting a robust approach. Zhang and Siu [32] discussed an optimal proportional reinsurance and investment problem using the criterion of maximizing utility function on terminal wealth. Zhang et al. [33] considered a Bayesian approach to incorporate parameter uncertainty in an optimal reinsurance and investment problem under a diffusion model for the surplus process of an insurer. Liu et al. [34] considered an optimal insurance-reinsurance problem in the presence of dynamic risk constraint and regime switching.

As we know, both the stochastic interest rates and inflation cannot be ignored for decision makers when dealing with long-term decision problems. Therefore, the incorporation of both stochastic interest rate and inflation makes our problems more practicable for the long-term decision making and this is the first contribution of our paper. The second contribution of our paper is that we establish a verification theorem for the solution using an approach which is not the same as the usual approach adopted for proving a verification theorem. In fact, unlike previous literature studies, the usual standard technical conditions required by the verification theorem for the solution of the optimal investment-reinsurance problem, such as the Lipschitz condition and the linear growth condition, are not satisfied by the model considered, which makes the proof of the verification theorem to be a challenging thing.

The rest of this paper is organized as follows. Section 2 presents the model dynamics and assumptions in the economy, finance, and insurance markets. The optimization problem of the insurer is formulated in Section 3. Furthermore, the closed-form solutions to the problem under the two stochastic interest rate models are derived by invoking the use of the HJB dynamic programming approach. In Section 4, the verification theorem for the solutions of the problem under the two models is established. Numerical comparison and analysis are presented in Section 5. The final section gives some concluding remarks. The proofs of some results are placed in an Appendix A.

\section{Model Dynamics and Assumptions}

To begin with, as usual, we describe uncertainties in the continuous-time economy, finance, and insurance markets as well as their information flows by a complete filtered probability space $(\Omega, \mathscr{F}, \mathbb{F}, p)$, where $\mathbb{F}=\left\{\mathscr{F}_{t}\right\}_{t \in[0, T]}$ is right continuous, $R$ is the complete filtration, and $R$ is a realworld probability. For simplicity, it is supposed that the filtration is "sufficiently large" so that all of the processes to be defined below are $\mathbb{F}$-adapted.

2.1. Price Index. The key economic variable we are intending to model is inflation. This is relevant to the optimal reinsurance and investment problem of an insurer since the decision making horizon of an insurer is usually long, say a decade or more, and inflation is a key economic factor which could lead to diminution of financial wealth of the insurer. Inflation can be measured by an inflation rate, which may be proxied by the annualized percentage change in a price index. Examples of a price index are consumer price indices and retail price indices. Incorporating inflation in studying optimal asset allocation problem has been considered in the literature. Some examples are Munk et al. [15], Pearson and Sun [14], Brennan and Xia [16], Douglas [35], George [36], and Fama and Gibbons [37]. It is usually assumed that a price index, which is used to proxy an inflation rate, is modeled by a stochastic process. Here, as in Munk et al. [15] and Yao et al. [38], it is supposed that the evolution of the nominal price index of a consumption good in an economy over time is governed by the following geometric Brownian motion with stochastic drift:

$$
\begin{aligned}
& \mathrm{d} \Pi(t)=\Pi(t)\left[I(t) \mathrm{d} t+\sigma_{0}(t) \mathrm{d} W_{0}(t)\right], \\
& \text { for all } t \in[0, T], \Pi(0)=\Pi_{0},
\end{aligned}
$$

where $\left\{W_{0}(t)\right\}_{t \in[0, T]}$ is a standard Brownian motion; $\sigma_{0}(t)$ is the volatility of the price index; and the stochastic drift $I(t)$ represents the instantaneous expected inflation rate whose evolution over time is assumed to be governed by the following time-dependent Ornstein-Uhlenbeck (OU) process:

$$
\mathrm{d} I(t)=\beta(t)[\alpha(t)-I(t)] \mathrm{d} t+\bar{\sigma}_{0}(t) \mathrm{d} W_{0}(t) .
$$

Here, $\alpha(t)$ describes the long-run mean of the expected inflation rate; $\beta(t)$ represents the degree of mean-reversion; and $\bar{\sigma}_{0}(t)$ is the volatility of the expected inflation rate. Consequently, the mean-reverting property of the expected inflation rate is incorporated in the model considered here. To simplify our discussion, it is assumed that $\sigma_{0}(t), \alpha(t), \beta(t)$, and $\bar{\sigma}_{0}(t)$ are deterministic and continuous functions of time $t \in[0, T]$. However, we adopt here a time-dependent OU process rather than the one in Munk et al. [15] and Yao et al. [38].

2.2. Financial Market. Assume that the financial market considered here consists of three assets: one savings account, one stock, and one zero-coupon bond with maturity $T_{1}>T$.

Let $\{B(t)\}_{t \in[0, T]}$ denote the price process of the savings account. Assume that the evolution of $\{B(t)\}_{t \in[0, T]}$ over time is determined by

$$
\mathrm{d} B(t)=r(t) B(t) \mathrm{d} t, \quad B(0)=1 .
$$

Here, $r(t)$ is the short-term interest rate, or the spot rate, whose evolution over time is governed by the following stochastic differential equation:

$$
\mathrm{d} r(t)=a(t) \mathrm{d} t+b \mathrm{~d} W_{1}(t), \quad t \in\left[0, T_{1}\right], r(0)=r_{0},
$$

where $b$ is a positive constant and $\left\{W_{1}(t)\right\}_{t \in[0, T]}$ is a standard Brownian motion. Following some explicit examples in Korn and Kraft [19], the Ho-Lee model and the Vasicek model are considered here, where $a(t)$ is, respectively, given by $a(t)=\widetilde{a}(t)+b \xi(t)$ and $a(t)=\theta(t)-\hat{b} r(t)+b \xi(t)$. Here, the risk premium $\xi(t)$ and parameter $\theta(t)$ are assumed to be deterministic and continuous functions of time $t \in[0, T]$.

Let $P\left(t, T_{1}\right)$ denote the price process of a zero-coupon bond with maturity $T_{1}>T$. Then, from Korn and Kraft [19], $P\left(t, T_{1}\right)$ satisfies the following stochastic differential equation: 


$$
\begin{aligned}
\mathrm{d} P\left(t, T_{1}\right) & =P\left(t, T_{1}\right)\left\{\left[r(t)+\xi(t) \sigma_{1}(t)\right] \mathrm{d} t+\sigma_{1}(t) \mathrm{d} W_{1}(t)\right\}, \\
P\left(0, T_{1}\right) & =P_{0}>0,
\end{aligned}
$$

where $r(t)$ is given by (4). In what follows, we shall write $P(t)$ for $P\left(t, T_{1}\right)$ to ease the notation. As shown in Korn and Kraft [19], the volatilities of the zero-coupon bond under the Ho-Lee model and Vasicek model are given by $\sigma(t)=-b\left(T_{1}-t\right)$ and $\sigma(t)=(b / \widehat{b})\left[\exp \left\{-\widehat{b}\left(T_{1}-t\right)\right\}-1\right]$, respectively.

Furthermore, it is supposed that the stock price process $\{S(t)\}_{t \in[0, T]}$ evolves over time according to the following geometric Brownian motion:

$$
\mathrm{d} S(t)=S(t)\left[\mu(t) \mathrm{d} t+\sigma_{2}(t) \mathrm{d} W_{2}(t)\right], \quad S(0)=S_{0},
$$

where $\left\{W_{2}(t)\right\}_{t \in[0, T]}$ is a standard Brownian motion and $\sigma_{2}(t)$ is the volatility of the stock at time $t$, where it is assumed that $\sigma_{2}(t)$ is a deterministic and continuous function of time $t$. As in Korn and Kraft [19], we decompose the appreciation rate $\mu(t)$ of the stock into the sum of a liquidity premium (LP) and a risk premium (RP):

$$
\mu(t)=\underbrace{r(t)}_{r(t)}+\underbrace{\mu(t)-r(t)}_{\mathrm{RP}} .
$$

Let $\tilde{\lambda}(t)$ denote the risk premium of the stock, i.e., $\tilde{\lambda}(t):=\mu(t)-r(t)$. Consequently, the price process of the stock can be rewritten as

$$
\mathrm{d} S(t)=S(t)\left\{[r(t)+\tilde{\lambda}(t)] \mathrm{d} t+\sigma_{2}(t) \mathrm{d} W_{2}(t)\right\} .
$$

2.3. Surplus Process. Let $\widetilde{R}(t)$ be the surplus of an insurance company at time $t \in[0, T]$. Since optimal long-term investment and reinsurance decision making of the insurance company are considered, it is relevant to incorporate the impact of inflation on the surplus of the insurance company. Consequently, it is supposed here that the increment of the surplus of the company depends on the price index described in Section 2.1. Specifically, without loss of generality, it is assumed that the surplus process of the company $\{\widetilde{R}(t)\}_{t \in[0, T]}$ evolves over time according to a diffusion approximation model with the impact of the price index being incorporated as follows:

$$
\mathrm{d} \widetilde{R}(t)=\Pi(t) c(t) \mathrm{d} t+\Pi(t) \sigma_{3}(t) \mathrm{d} W_{3}(t),
$$

where $\left\{W_{3}(t)\right\}_{t \in[0, T]}$ is a standard Brownian motion and $c(t)$ and $\sigma_{3}(t)$ are deterministic and continuous function of time $t \in[0, T]$ with $c(t)>0$. Note that $c(t)$ and $\sigma_{3}(t)$ may be, respectively, interpreted as the real premium rate of the insurer and the risk attributed to uncertainty about future insurance liabilities. See, for example, Jan [39], Zeng and Li [40], Højgaard and Taksar [41], and Michael and Markussen [42] for more details about diffusion approximation models to surplus processes of insurance companies.

Remark 1. Note that $d \widetilde{R}(t)$ denotes the instantaneous increment that incorporates the impact of price index. Equation (9) indicates that the instantaneous increment changes in the surplus depends on the price index $\Pi(t)$. In other words, the real instantaneous increment change is given by $c(t) \mathrm{d} t+\sigma(t) \mathrm{d} W_{3}(t)$.

Let $u(t)$ be the proportional reinsurance retention level adopted by the insurance company at time $t$, where $u(t) \in[0, \infty)$. Assume, for simplicity, that the safety loadings of the insurance company and the reinsurance company are the same. Consequently, the surplus process of insurance company $\{R(t)\}_{t \in[0, T]}$ after acquiring the proportional reinsurance is given by

$$
\mathrm{d} R(t)=\Pi(t) u(t) c(t) \mathrm{d} t+\Pi(t) u(t) \sigma_{3}(t) \mathrm{d} W_{3}(t) .
$$

Lastly, we assume that the stochastic interest rate, the bond price, the price index, and the expected inflation rate could be correlated. Without loss of generality, we suppose $\operatorname{Cov}\left(W_{1}(t), W_{0}(t)\right)=\rho t, \rho \in(-1,1)$. Also assume that $\left\{W_{2}(t)\right\}_{t \in[0, T]}$ and $\left\{W_{3}(t)\right\}_{t \in[0, T]}$ are independent Brownian motions which are independent of $\left\{W_{1}(t)\right\}_{t \in[0, T]}$ and $\left\{W_{0}(t)\right\}_{t \in[0, T]}$. If we suppose the interest rate or the price index is correlated with the stock, there will be an additional mixed partial derivative term in HJB equation, but it will not affect the method used in the remainder of the paper.

2.4. Wealth Process. Suppose that the insurer is allowed to continuously purchase proportional reinsurance and invests all of his (or her) wealth in the financial market over the time $[0, T]$ with $T<T_{1}$. This assumption is an idealisation in the continuous-time modeling set up here. In practice, an insurer may only be able to acquire reinsurance treaties in discrete time periods and there are frictional or transaction costs when the insurer invests in the security market.

Let $\pi_{1}(t)$ and $\pi_{2}(t)$ be the proportions of the total wealth invested in the bond and stock at time $t$, respectively. Suppose $u(t)$ denotes the proportional reinsurance retention level at time $t$. Accordingly, $1-\pi_{1}(t)-\pi_{2}(t)$ is the proportion of the total wealth invested in the saving account. If we denote by $\widetilde{X}(t)$ the wealth of the insurer at time $t$ after adopting the reinsurance and investment, then we have

$$
\begin{aligned}
\mathrm{d} \widetilde{X}(t)= & \pi_{1}(t) \widetilde{X}(t) \frac{\mathrm{d} P(t)}{P(t)}+\pi_{2}(t) \tilde{X}(t) \frac{\mathrm{d} S(t)}{S(t)} \\
& +\left(1-\pi_{1}(t)-\pi_{2}(t)\right) \widetilde{X}(t) \frac{\mathrm{d} B(t)}{B(t)}+\Pi(t) u(t) c(t) \mathrm{d} t \\
& +\Pi(t) u(t) \sigma_{3}(t) \mathrm{d} W_{3}(t) \\
= & \widetilde{X}(t)\left\{\left[r(t)+\pi_{1}(t) \xi(t) \sigma_{1}(t)+\pi_{2}(t) \widetilde{\lambda}(t)\right] \mathrm{d} t\right. \\
& \left.+\pi_{1}(t) \sigma_{1}(t) \mathrm{d} W_{1}(t)+\pi_{2}(t) \sigma_{2}(t) \mathrm{d} W_{2}(t)\right\} \\
& +\Pi(t) u(t) c(t) \mathrm{d} t+\Pi(t) u(t) \sigma_{3}(t) \mathrm{d} W_{3}(t) .
\end{aligned}
$$

In Section 2.3, the nominal price index of the consumption good in the economy at time $t$ is denoted by $\Pi(t)$. 
The real price of an asset in the economy over a long time is determined by deflating its nominal value with the price index $\Pi(t)$. The real wealth of the insurer, which adjusts for the impact of inflation, is given by $X(t)=\widetilde{X}(t) / \Pi(t)$. Then, applying the Itô formula (see, for example, Karatzas and Shreve [43]) gives

$$
\begin{aligned}
\mathrm{d} X(t)= & \left\{X ( t ) \left[r(t)+\sigma_{0}^{2}(t)-I(t)+\left(\xi(t) \sigma_{1}(t)\right.\right.\right. \\
& \left.\left.\left.-\rho \sigma_{1}(t) \sigma_{0}(t)\right) \pi_{1}(t)+\tilde{\lambda}(t) \pi_{2}(t)\right]+u(t) c(t)\right\} \mathrm{d} t \\
& +u(t) \sigma_{3}(t) \mathrm{d} W_{3}(t)+X(t) \pi_{1}(t) \sigma_{1}(t) \mathrm{d} W_{1}(t) \\
& +X(t) \pi_{2}(t) \sigma_{2}(t) \mathrm{d} W_{2}(t)-X(t) \sigma_{0}(t) \mathrm{d} W_{0}(t)
\end{aligned}
$$

and the initial value $X(0)=(\widetilde{X}(0) / \Pi(0))=X_{0}$.

In what follows, we take $\eta(t)=\xi(t)-\rho \sigma_{0}(t)$. With a slight abuse of the notation, we use $\lambda(t)$ to denote $\widetilde{\lambda}(t) / \sigma_{2}(t)$. Consequently, we can rewrite the wealth process as

$$
\begin{aligned}
\mathrm{d} X(t)= & \left\{X ( t ) \left[r(t)+\sigma_{0}^{2}(t)-I(t)+\sigma_{1}(t) \eta(t) \pi_{1}(t)\right.\right. \\
& \left.\left.+\lambda(t) \sigma_{2}(t) \pi_{2}(t)\right]+u(t) c(t)\right\} \mathrm{d} t \\
& +u(t) \sigma_{3}(t) \mathrm{d} W_{3}(t)+X(t) \pi_{1}(t) \sigma_{1}(t) \mathrm{d} W_{1}(t) \\
& +X(t) \pi_{2}(t) \sigma_{2}(t) \mathrm{d} W_{2}(t)-X(t) \sigma_{0}(t) \mathrm{d} W_{0}(t)
\end{aligned}
$$

with $X(0)=X_{0}$.

Next, we give the definition of an admissible control.

Definition 1. A strategy $\tilde{\pi}(t)=\left(\pi_{1}(t), \pi_{2}(t), u(t)\right)$ is said to be admissible if $(1) \pi_{1}(t), \pi_{2}(t)$, and $u(t)$ are progressively measurable processes; (2) $\pi_{1}(t)$ and $\pi_{2}(t)$ are bounded; (3) $u(t) \geq 0$; (4) $\tilde{\pi}(t)$ leads to a positive wealth process. We denote $\Theta$ by the set of all admissible controls.

\section{Maximizing the Expected Power Utility}

In this section, we first present the optimal investment and reinsurance problem of an insurer as an utility maximization problem, where the insurer aims to select an investmentreinsurance mix to maximize the expected power utility on terminal wealth. Then, using the standard dynamic programming approach (see, for example, Wendell [44] and Fleming and Soner [45]), we derive the Hamilton-JacobiBellman (HJB) equation governing the value function of the utility maximization problem.

Suppose that the insurer's preference is described by a non-log hyperbolic absolute risk aversion (HARA) utility function $U(x)=(1 / p) x^{p}, 0<p<1, x>0$ and that the insurer wishes to maximize the expected utility of terminal wealth at time $T$. For any strategy $\tilde{\pi} \in \Theta$, the expected power utility of the insurer at time $T$, denoted by $V_{\tilde{\pi}}(t, x, r, I)$, is given by

$$
V_{\widetilde{\pi}}(t, x, r, I)=E\left\{\frac{1}{p}\left(X_{T}^{\tilde{\pi}}\right)^{p} \mid X_{t}=x, r_{t}=r, I_{t}=I\right\},
$$

where the dynamics of $\left\{X_{t}^{\tilde{\pi}}\right\}_{t \in[0, T]},\left\{r_{t}\right\}_{t \in[0, T]}$, and $\left\{I_{t}\right\}_{t \in[0, T]}$ are governed by the following three stochastic differential equations:

$$
\left\{\begin{array}{l}
\mathrm{d} X(t)=\left\{X(t)\left[r(t)+\sigma_{0}^{2}(t)-I(t)+\sigma_{1}(t) \eta(t) \pi_{1}(t)+\lambda(t) \sigma_{2}(t) \pi_{2}(t)\right]+u(t) c(t)\right\} \mathrm{d} t \\
+u(t) \sigma_{3}(t) \mathrm{d} W_{3}(t)+X(t) \pi_{1}(t) \sigma_{1}(t) \mathrm{d} W_{1}(t)+X(t) \pi_{2}(t) \sigma_{2}(t) \mathrm{d} W_{2}(t)-X(t) \sigma_{0}(t) \mathrm{d} W_{0}(t) \\
\mathrm{d} r(t)=a(t) \mathrm{d} t+b \mathrm{~d} W_{1}(t) \\
\mathrm{d} I(t)=\beta(t)[\alpha(t)-I(t)] \mathrm{d} t+\bar{\sigma}_{0}(t) \mathrm{d} W_{0}(t),
\end{array}\right.
$$

Our goal is to find the value function:

$$
V(t, x, r, I)=\sup _{\tilde{\pi} \in \Theta} V_{\widetilde{\pi}}(t, x, r, I),
$$

and the optimal strategy $\tilde{\pi}^{*}=\left(\pi_{1}^{*}, \pi_{2}^{*}, u^{*}\right)$ such that

$$
V(t, x, r, I)=V_{\tilde{\pi}^{*}}(t, x, r, I) .
$$

Using standard arguments in dynamic programming, the value function satisfies the following HJB equation with the corresponding terminal condition as follows: 


$$
\begin{aligned}
0= & V_{t}+V_{x}\left[r-I+\sigma_{0}^{2}(t)\right] x+\frac{1}{2} V_{x x} \sigma_{0}^{2}(t) x^{2}+a(t) V_{r} \\
& +\frac{1}{2} b^{2} V_{r r}+\beta(t)[\alpha(t)-I] V_{I} \\
& +\frac{1}{2} \bar{\sigma}_{0}^{2}(t) V_{I I}-\rho \sigma_{0}(t) b x V_{x r}-\bar{\sigma}_{0}(t) \sigma_{0}(t) x V_{x I} \\
& +b \bar{\sigma}_{0}(t) \rho V_{r I}+\sup _{\pi_{1} \in R}\left\{\frac{1}{2} V_{x x} \sigma_{1}^{2}(t) x^{2} \pi_{1}^{2}+\left[V_{x} \sigma_{1}(t) \eta(t) x\right.\right. \\
& -V_{x x} \rho \sigma_{1}(t) \sigma_{0}(t) x^{2}+V_{x r} b \sigma_{1}(t) x \\
& \left.\left.+V_{x I} \bar{\sigma}_{0}(t) \sigma_{1}(t) \rho x\right] \pi_{1}\right\} \\
& +\sup _{\pi_{2} \in R}\left\{\frac{1}{2} V_{x x} \sigma_{2}^{2}(t) x^{2} \pi_{2}^{2}+V_{x} \lambda(t) \sigma_{2}(t) x \pi_{2}\right\} \\
& +\sup _{u \in[0, \infty)}\left\{\frac{1}{2} V_{x x} \sigma_{3}^{2}(t) u^{2}+c(t) V_{x} u\right\},
\end{aligned}
$$$$
V(T, x, I, r)=\frac{1}{p} x^{p}
$$

In what follows, we first present a solution to HJB equation (18) with terminal condition (19). Suppose that HJB equation (18) with terminal condition (19) has a classical solution $G$ satisfying the conditions that $G_{x}>0$ and that $G_{x x}<0$. Furthermore, we assume that the solution $G$ has the following form:

$$
G(t, x, r, I)=g(t, r, I) \frac{x^{p}}{p},
$$

where $g(t, r, I)$ is a function with the terminal condition $g(T, r, I)=1$ for all $I$ and $r$, and it will be determined in the sequel.

Differentiating the left hand side of equation (18) with respect to $\pi_{1}, \pi_{2}$, and $u$ and setting the derivatives equal to 0 yield the following candidate optimal controls:

$$
\begin{aligned}
& \pi_{1}^{*}(t)=-\frac{\eta(t)}{\sigma_{1}(t)} \frac{G_{x}}{x G_{x x}}-\frac{\bar{\sigma}_{0}(t) \rho}{\sigma_{1}(t)} \frac{G_{I x}}{x G_{x x}}-\frac{b}{\sigma_{1}(t)} \frac{G_{x r}}{x G_{x x}}+\frac{\sigma_{0}(t) \rho}{\sigma_{1}(t)} \\
& \pi_{2}^{*}(t)=-\frac{G_{x}}{x G_{x x}} \frac{\lambda(t)}{\sigma_{2}(t)} \\
& u^{*}(t)=-\frac{G_{x}}{G_{x x}} \frac{c(t)}{\sigma_{3}^{2}(t)}
\end{aligned}
$$

Note that for the function $G$, we have

$$
\begin{aligned}
G_{t} & =g_{t} \frac{x^{p}}{p}, \\
G_{x} & =g x^{p-1}, \\
G_{x x} & =g(p-1) x^{p-2}, \\
G_{r} & =g_{r} \frac{x^{p}}{p}, \\
G_{r r} & =g_{r r} \frac{x^{p}}{p}, \\
G_{I} & =g_{I} \frac{x^{p}}{p}, \\
G_{I I} & =g_{I I} \frac{x^{p}}{p}, \\
G_{x r} & =g_{r} x^{p-1}, \\
G_{x I} & =g_{I} x^{p-1}, \\
G_{r I} & =g_{r I} \frac{x^{p}}{p} .
\end{aligned}
$$
leads to

Consequently, substituting the above equations into (18)

$$
\begin{aligned}
0= & \frac{g_{t}}{p}+g\left(r+\sigma_{0}^{2}-I\right)+\frac{1}{2} \sigma_{0}^{2} g(p-1)+a g_{r} \frac{1}{p}+\frac{1}{2} b^{2} \frac{g_{r r}}{p} \\
& +\frac{g_{I}}{p} \beta(\alpha-I)+\frac{1}{2} \bar{\sigma}_{0}^{2} \frac{g_{I I}}{p}-\sigma_{0} \bar{\sigma}_{0} g_{I}-g_{r} \rho \sigma_{0} b+\frac{g_{I r}}{p} b \rho \bar{\sigma}_{0} \\
& -\frac{g}{p-1} \frac{\eta^{2}}{2}-\frac{1}{2} g(p-1) \sigma_{0}^{2} \rho^{2}-\frac{g_{I}^{2}}{g(p-1)} \frac{\bar{\sigma}_{0}^{2} \rho^{2}}{2} \\
& -\frac{g_{r}^{2}}{g(p-1)} \frac{b^{2}}{2}+g \eta \sigma_{0} \rho-\frac{g_{I}}{p-1} \eta \rho \bar{\sigma}_{0}-\frac{g_{r}}{p-1} b \eta \\
& +g_{r} b \sigma_{0} \rho+g_{I} \bar{\sigma}_{0} \sigma_{0} \rho^{2}-\frac{g_{r} g_{I}}{g(p-1)} \bar{\sigma}_{0} \rho b-\frac{g}{p-1} \frac{\lambda^{2}}{2} \\
& -\frac{g}{p-1} \frac{c^{2}}{2 \sigma_{3}^{2}} .
\end{aligned}
$$

Consider the following trial solution:

$$
g(t, r, I)=f(t) e^{k(t) r+z(t) I},
$$

where $f(t), k(T)$, and $z(t)$ are the functions of time $t$ with their respective terminal values: $f(T)=1, k(T)=0$, and $z(T)=0$. Let $\Delta:=k(t) r+z(t) I$. Then, 


$$
\begin{aligned}
g_{t} & =f^{\prime} e^{\Delta}+f e^{\Delta}\left(k^{\prime} r+z^{\prime} I\right), \\
g_{r} & =f k e^{\Delta}, \\
g_{I} & =f z e^{\Delta}, \\
g_{I r} & =f k z e^{\Delta}, \\
g_{r r} & =f k^{2} e^{\Delta}, \\
g_{I I} & =f z^{2} e^{\Delta} .
\end{aligned}
$$

Substituting them into (25) yields

$$
\begin{aligned}
0= & {\left[\frac{k^{\prime}}{p}+1\right] f r+\left[\frac{z^{\prime}}{p}-\frac{\beta z}{p}-1\right] f I+\frac{f^{\prime}}{p} } \\
& +\left\{\sigma_{0}^{2}+\frac{1}{2}(p-1) \sigma_{0}^{2}+\frac{b^{2} k^{2}}{2 p}+\frac{\alpha \beta}{p} z+\frac{\bar{\sigma}_{0}^{2} z^{2}}{2 p}\right. \\
& -\bar{\sigma}_{0} \sigma_{0} z-k \rho \sigma_{0} b+\frac{b \rho \bar{\sigma}_{0}}{p} k z-\frac{\eta^{2}}{2(p-1)}-\frac{p-1}{2} \rho^{2} \sigma_{0}^{2} \\
& -\frac{\rho^{2} \bar{\sigma}_{0}^{2}}{2(p-1)} z^{2}-\frac{b^{2}}{2(p-1)} k^{2}+\sigma_{0} \rho \eta-\frac{\eta \rho \bar{\sigma}_{0}}{p-1} z \\
& -\frac{\eta b}{p-1} k+\bar{\sigma}_{0} \sigma_{0} \rho^{2} z+\sigma_{0} b \rho k-\frac{\bar{\sigma}_{0} \rho b}{p-1} k z-\frac{\lambda^{2}}{2(p-1)} \\
& \left.-\frac{c^{2}}{2 \sigma_{3}^{2}(p-1)}\right\} f+\frac{a k}{p} f .
\end{aligned}
$$

Due to the different forms of $a(t)$ for the Ho-Lee model and Vasicek model, we solve the above differential equation separately.

\subsection{Ho-Lee Model. Let}

$$
\begin{aligned}
h(t):= & \sigma_{0}^{2}+\frac{1}{2}(p-1) \sigma_{0}^{2}+\frac{(\tilde{a}+b \xi) k}{p}+\frac{b^{2} k^{2}}{2 p}+\frac{\alpha \beta}{p} z+\frac{\bar{\sigma}_{0}^{2} z^{2}}{2 p} \\
& -\bar{\sigma}_{0} \sigma_{0} z-k \rho \sigma_{0} b+\frac{b \rho \bar{\sigma}_{0}}{p} k z-\frac{\eta^{2}}{2(p-1)}-\frac{p-1}{2} \rho^{2} \sigma_{0}^{2} \\
& -\frac{\rho^{2} \bar{\sigma}_{0}^{2}}{2(p-1)} z^{2}-\frac{b^{2}}{2(p-1)} k^{2}+\sigma_{0} \rho \eta-\frac{\eta \rho \bar{\sigma}_{0}}{p-1} z \\
& -\frac{\eta b}{p-1} k+\bar{\sigma}_{0} \sigma_{0} \rho^{2} z+\sigma_{0} b \rho k-\frac{\bar{\sigma}_{0} \rho b}{p-1} k z \\
& -\frac{\lambda^{2}}{2(p-1)}-\frac{c^{2}}{2 \sigma_{3}^{2}(p-1)} .
\end{aligned}
$$

Suppose that $k(t), z(t)$, and $f(t)$ are the solutions to the following ordinary differential equations:

$$
\begin{aligned}
& \left\{\begin{array}{l}
k^{\prime}(t)=-p, \\
k(T)=0,
\end{array}\right. \\
& \left\{\begin{array}{l}
z^{\prime}(t)-\beta(t) z(t)-p=0, \\
z(T)=0,
\end{array}\right. \\
& \left\{\begin{array}{l}
f^{\prime}(t)+p h(t) f(t)=0, \\
f(T)=1 .
\end{array}\right.
\end{aligned}
$$

Note that for the Ho-Lee model, $a(t):=\tilde{a}(t)+b \xi(t)$. Therefore, we can rewrite (28) as

$$
0=\left[\frac{k^{\prime}}{p}+1\right] f r+\left[\frac{z^{\prime}}{p}-\frac{\beta z}{p}-1\right] f I+\frac{f^{\prime}}{p}+h(t) f .
$$

It can be seen that the functions $k(t), z(t)$, and $f(t)$ determined by (30)-(32) satisfy equation (33). Using standard theory of ordinary differential equations, explicit expressions for $k(t), z(t)$, and $f(t)$ are obtained as follows:

$$
\left\{\begin{array}{l}
k(t)=p(T-t), \\
z(t)=-p e^{\int_{0}^{t} \beta(s) \mathrm{d} s} \int_{t}^{T} e^{-\int_{0}^{s} \beta(v) \mathrm{d} v} \mathrm{~d} s, \\
f(t)=e^{-p \int_{t}^{T} h(s) \mathrm{d} s} .
\end{array}\right.
$$

Combining (20), (26), and (34), an explicit solution to HJB (18) with terminal condition (19) is obtained as follows:

$$
G(t, x, r, I)=\frac{1}{p} \exp \left\{-p \int_{t}^{T} h(s) \mathrm{d} s+k(t) r+z(t) I\right\} x^{p},
$$

where $k(t)$ and $z(t)$ are given by (34). Now, from equations (21)-(23), the following expressions for the candidate optimal controls are obtained:

$$
\begin{aligned}
\pi_{1}^{*}(t)= & -\frac{\eta(t)}{\sigma_{1}(t)} \frac{1}{p-1}-\frac{b}{\sigma_{1}(t)} \frac{p}{p-1}(T-t)+\frac{\rho \sigma_{0}(t)}{\sigma_{1}(t)} \\
& -\frac{\rho \bar{\sigma}_{0}(t)}{\sigma_{1}(t)} \frac{p}{p-1} e^{\int_{0}^{t} \beta(s) \mathrm{d} s} \int_{t}^{T} e^{-\int_{0}^{s} \beta(v) \mathrm{d} v} \mathrm{~d} s, \\
\pi_{2}^{*}(t)= & -\frac{\lambda(t)}{\sigma_{2}(t)} \frac{1}{p-1}, \\
u^{*}(t)= & -\frac{c(t)}{\sigma_{3}^{2}(t)} \frac{1}{p-1} x .
\end{aligned}
$$


3.2. Vasicek Model. Let

$$
\begin{aligned}
\widetilde{h}(t):= & \sigma_{0}^{2}+\frac{1}{2}(p-1) \sigma_{0}^{2}+\frac{(\theta+b \xi) k}{p}+\frac{b^{2} k^{2}}{2 p}+\frac{\alpha \beta}{p} z+\frac{\bar{\sigma}_{0}^{2} z^{2}}{2 p} \\
& -\bar{\sigma}_{0} \sigma_{0} z-k \rho \sigma_{0} b+\frac{b \rho \bar{\sigma}_{0}}{p} k z-\frac{\eta^{2}}{2(p-1)}-\frac{p-1}{2} \rho^{2} \sigma_{0}^{2} \\
& -\frac{\rho^{2} \bar{\sigma}_{0}^{2}}{2(p-1)} z^{2}-\frac{b^{2}}{2(p-1)} k^{2}+\sigma_{0} \rho \eta-\frac{\eta \rho \bar{\sigma}_{0}}{p-1} z \\
& -\frac{\eta b}{p-1} k+\bar{\sigma}_{0} \sigma_{0} \rho^{2} z+\sigma_{0} b \rho k-\frac{\bar{\sigma}_{0} \rho b}{p-1} k z-\frac{\lambda^{2}}{2(p-1)} \\
& -\frac{c^{2}}{2 \sigma_{3}^{2}(p-1)} .
\end{aligned}
$$

Suppose that $\widetilde{k}(t), \widetilde{z}(t)$, and $\widetilde{f}(t)$ are the solutions to the following ordinary differential equations:

$$
\begin{aligned}
& \left\{\begin{array}{l}
\tilde{k}^{\prime}(t)-\widehat{b} k(t)+p=0, \\
\tilde{k}(T)=0,
\end{array}\right. \\
& \left\{\begin{array}{l}
\widetilde{z}^{\prime}(t)-\beta(t) \widetilde{z}(t)-p=0, \\
\widetilde{z}(T)=0,
\end{array}\right. \\
& \left\{\begin{array}{l}
\tilde{f}^{\prime}(t)+p \tilde{h}(t) \tilde{f}(t)=0, \\
\tilde{f}(T)=1 .
\end{array}\right.
\end{aligned}
$$

Note that for the Vasicek model, $a(t)=\theta(t)-\widehat{b} r(t)+b \xi(t)$, and so (28) is equivalent to

$$
0=\left[\frac{k^{\prime}}{p}-\frac{\widehat{b}}{p} k+1\right] f r+\left[\frac{z^{\prime}}{p}-\frac{\beta z}{p}-1\right] f I+\frac{f^{\prime}}{p}+\tilde{h} f .
$$

It can be seen that the functions $\tilde{k}(t), \tilde{z}(t)$, and $\tilde{f}(t)$ determined by (38)-(40) satisfy equation (41). Again using the standard theory of ordinary differential equations, the following explicit expressions for $\widetilde{k}(t), \widetilde{z}(t)$, and $\widetilde{f}(t)$ under the Vasicek model are obtained:

$$
\left\{\begin{array}{l}
\widetilde{k}(t)=\frac{p}{\widehat{b}}[1-\exp \{\widehat{b}(t-T)\}] \\
\tilde{z}(t)=-p e^{\int_{0}^{t} \beta(s) \mathrm{d} s} \int_{t}^{T} e^{-\int_{0}^{s} \beta(v) \mathrm{d} v} \mathrm{~d} s \\
\tilde{f}(t)=e^{-p \int_{t}^{T} \widetilde{h}(s) d s} .
\end{array}\right.
$$

Combining (20), (26), and (42), an explicit solution for HJB equation (18) with terminal condition (19) under the Vasicek model is obtained as follows:

$$
G(t, x, r, I)=\frac{1}{p} \exp \left\{-p \int_{t}^{T} \widetilde{h}(s) \mathrm{d} s+\widetilde{k}(t) r+\widetilde{z}(t) I\right\} x^{p},
$$

where $\widetilde{k}(t)$ and $\widetilde{z}(t)$ are given by (42). Now, from equations (21)-(23), the following expressions for the candidate optimal controls under the Vasicek model are obtained:

$$
\left\{\begin{array}{l}
\pi_{1}^{*}(t)=-\frac{\eta(t)}{\sigma_{1}(t)} \frac{1}{p-1}-\frac{1}{\sigma_{1}(t)} \frac{p}{p-1}\left[1-e^{b(t-T)}\right] \\
-\frac{\rho \bar{\sigma}_{0}(t)}{\sigma_{1}(t)} \frac{p}{p-1} e^{\int_{0}^{t} \beta(s) \mathrm{d} s} \int_{t}^{T} e^{-\int_{0}^{s} \beta(v) \mathrm{d} v} \mathrm{~d} s+\frac{\rho \sigma_{0}(t)}{\sigma_{1}(t)} \\
\pi_{2}^{*}(t)=-\frac{\lambda(t)}{\sigma_{2}(t)} \frac{1}{p-1} \\
u^{*}(t)=-\frac{c(t)}{\sigma_{3}^{2}(t)} \frac{1}{p-1} x
\end{array}\right.
$$

\section{Verification Theorem}

A verification theorem for the solution to HJB (18) derived in the previous section is presented for the two stochastic interest rate models. The verification theorem states that the solution of HJB (18) is the value of the optimal control problem of the insurer and the candidate optimal control is an optimal control.

Due to the presence of $r(t) X(t)$ and $I(t) X(t)$ in the wealth process of (13), the usual verification theorem which requires Lipschitz and linear growth conditions is not applicable in our situation. Inspired by the methods used by Kraft [11], Li and Wu [20], and Kraft [21], we need to some uniform integrability of $\left\{G\left(\tau_{n}, X_{\tau_{n}}^{*}, I\left(\tau_{n}\right), r\left(\tau_{n}\right)\right)\right\}_{n \in N}$, where $\left\{X_{t}^{*}\right\}$ is the optimal wealth process and $\left\{\tau_{n}\right\}_{n \in N}$ is a sequence of stopping times which is bounded above by $T$.

Lemma 1. Let $\left\{X_{t}^{*}\right\}$ denote the respective optimal wealth process. Then,

(1) Ho-Lee model: suppose $G$ and $\tilde{\pi}^{*}(t)=\left(\pi_{1}^{*}(t), \pi_{2}^{*}(t), u^{*}(t)\right)$ are given by (35) and (36), respectively. Then, the sequence $\left\{G\left(\tau_{n}, X_{\tau_{n}}^{*}, I\left(\tau_{n}\right), r\left(\tau_{n}\right)\right)\right\}_{n \in N}$ is uniformly integrable for all sequences of stopping times $\left\{\tau_{n}\right\}_{n \in N}$ with bound $T$

(2) Vasicek model: suppose $G$ and $\tilde{\pi}^{*}(t)=\left(\pi_{1}^{*}(t), \pi_{2}^{*}(t), u^{*}(t)\right)$ are given by (43) and (44), respectively. $\left\{G\left(\tau_{n}, X_{\tau_{n}}^{*}, I\left(\tau_{n}\right), r\left(\tau_{n}\right)\right)\right\}_{n \in N}$ is uniformly integrable for all sequences of stopping times $\left\{\tau_{n}\right\}_{n \in N}$ with bound $T$ 
The main idea of proving the above lemma is from Kraft [11], $\mathrm{Li}$ and $\mathrm{Wu}$ [20], and Kraft [21], but there still exist some details different from these references. Therefore, we provide the proof in the appendix for the sake of completeness. Based on the uniform integrability property, we can prove the verification theorem.

Theorem 1 (verification theorem for two models)

(1) Ho-Lee model: Suppose $G(t, x, r, I)$ is defined by (35), then for any $\tilde{\pi}(t)=\left(\pi_{1}(t), \pi_{2}(t), u(t)\right) \in \Theta$, we have

$$
E_{t, x, r, I}\left[\frac{1}{p}\left(X_{T}^{\tilde{\pi}}\right)^{p}\right] \leq G(t, x, r, I) .
$$

Furthermore, the candidate optimal control $\tilde{\pi}^{*}$ defined by (36) is indeed the optimal control and

$$
V(t, x, r, I)=E_{t, x, r, I}\left[\frac{1}{p}\left(X_{T}^{\tilde{\pi}^{*}}\right)^{p}\right]=G(t, x, r, I) .
$$

(2) Vasicek model: Suppose $G(t, x, r, I)$ is defined by (43), then for any $\tilde{\pi}(t)=\left(\pi_{1}(t), \pi_{2}(t), u(t)\right) \in \Theta$, we have

$$
E_{t, x, r, I}\left[\frac{1}{p}\left(X_{T}^{\tilde{\pi}}\right)^{p}\right] \leq G(t, x, r, I) .
$$

Furthermore, the candidate optimal control $\tilde{\pi}^{*}$ defined by (44) is indeed the optimal control and

$$
V(t, x, r, I)=E_{t, x, r, I}\left[\frac{1}{p}\left(X_{T}^{\widetilde{\pi}^{*}}\right)^{p}\right]=G(t, x, r, I) .
$$

Proof. By Lemma 1, we know that $G(t, x, r, I)$ and $\tilde{\pi}^{*}$ satisfy the so-called "property U” of Definition 4.2 given by Kraft [21]. Therefore, applying the method used in Theorem 4.1 by Kraft [21] leads to the desired results of the theorem.

\section{Numerical Analysis}

In this section, we shall present numerical analysis for the optimal strategies under the two stochastic interest rate models. For simplicity, we assume the parameters are constant over time interval $t \in[0, T]$. Furthermore, we take some hypothetical values: $T=80, T_{1}=120, \eta=0.0606$, $b=0.05, \rho=-0.06$ and $\beta=0.02, \sigma_{0}=0.01$, and $\bar{\sigma}_{0}=0.026$.

The parameter $p, 0<p<1$, in the utility function represents the degree of risk aversion. For example, the more risk averse the investor is, the larger the parameter is. Figures 1 and 2 depict the changes in the proportion of the wealth invested in the bond for insurers having different attitudes toward risk, say different values of the parameter of $p$. From Figures 1 and 2, it can be seen that under each of the two stochastic interest models, say the Ho-Lee model and the Vasicek model, the insurer gradually increases the optimal proportion invested in

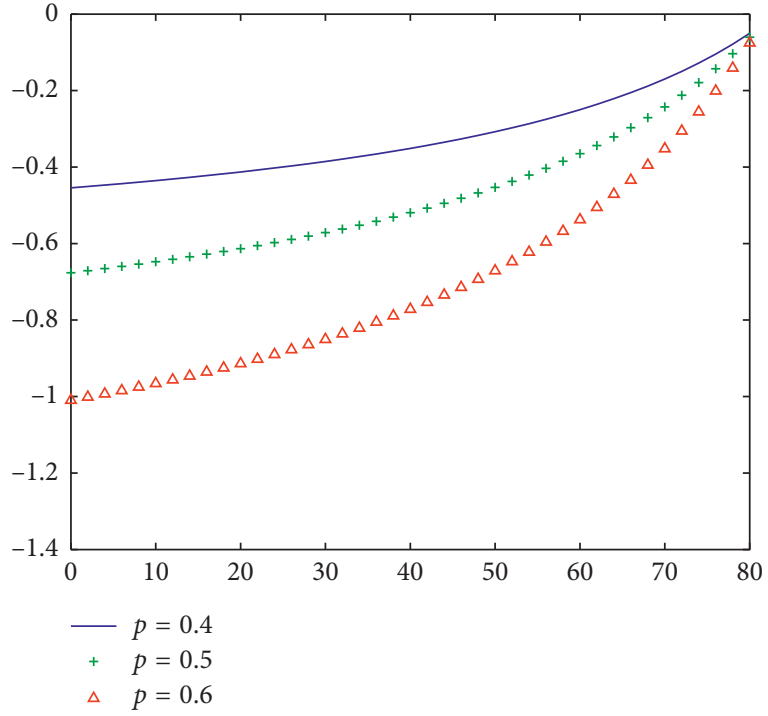

Figure 1: Case of the Ho-Lee model.

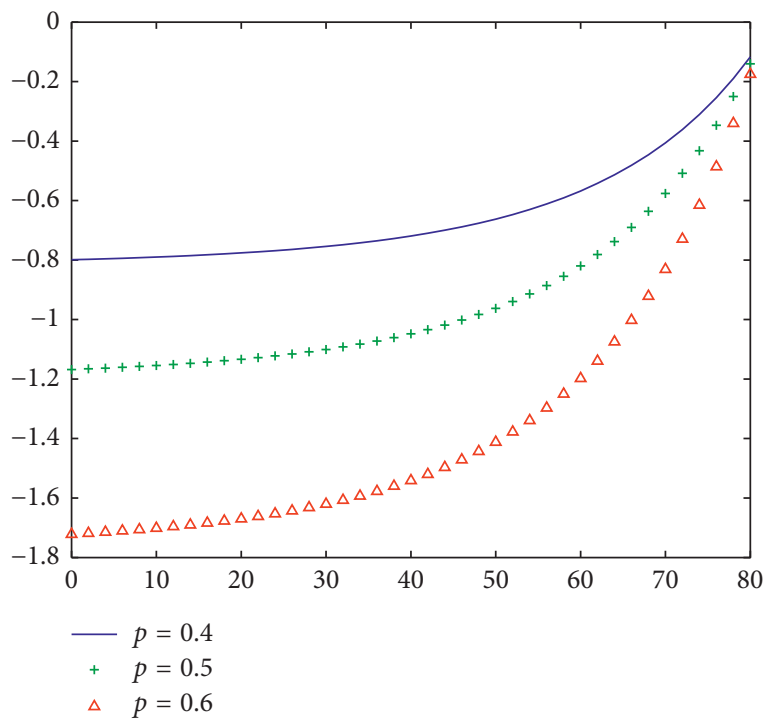

Figure 2: Case of the Vasicek model.

bond as time passes by. On the other hand, the two figures reveal that an investor who is more risk averse will invest less amount of money in the bond than the one who is less risk averse.

Figure 3 provides a comparison for the optimal policies of the insurer under the Ho-Lee model and the Vasicek model. In this case, we choose $p=0.5$, which represents a certain degree of risk aversion of the insurer. From this figure, it can be seen that if the Ho-Lee model is used to describe the stochastic interest rate, more money is invested in the bond than when the stochastic interest rate is modeled by the Vasicek model. The main feature that is described by the Vasicek model is that the interest rate will revert to a certain long-run mean level. Consequently, the numerical results reveal that the mean-reverting property of the 


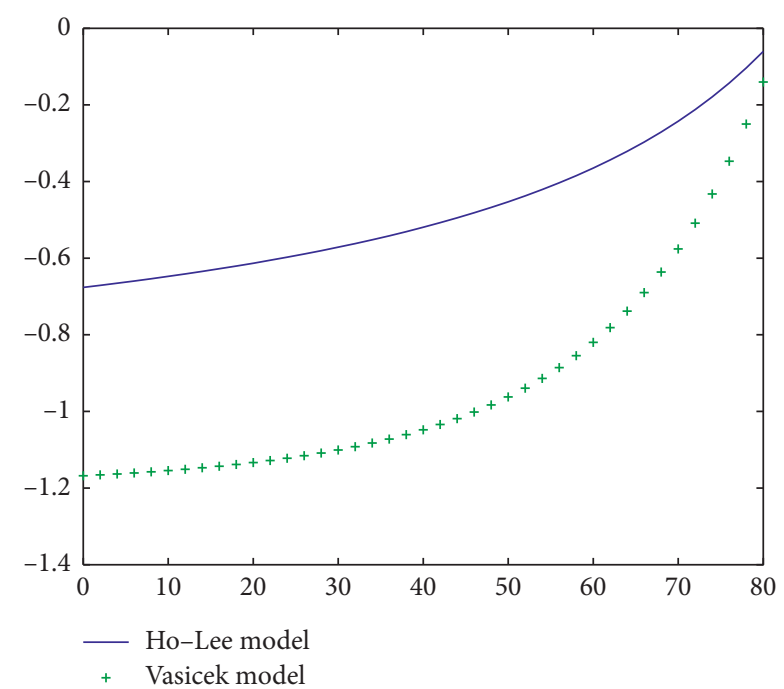

Figure 3: Comparison of the Ho-Lee model and Vasicek model on the position of bond.

stochastic interest rate may lead to a reduction in the optimal amount of money invested in the bond by the insurer.

\section{Conclusion}

The contribution of the present paper rests on the incorporation of both stochastic interest rate and inflation on long-term decision making of an insurance company. Specifically, the Ho-Lee model and the Vasicek model are employed to describe the stochastic interest rate. A challenging aspect of the problem is that certain standard technical conditions, such as the Lipschitz condition and the linear growth condition, which are required in a verification theorem for the solution of the optimal investment-reinsurance problem, are not satisfied by the model considered. Here, we establish a verification theorem for the solution using an approach which is not the same as the usual approach adopted for proving a verification theorem. Furthermore, a closed-form solution to the optimal reinsurance-investment problem is obtained for each of the two stochastic interest rate models. This may make the implementation of the optimal solutions easier. To shed light on understanding some implications of the optimal solutions, numerical analysis and comparison for the optimal solutions are provided to illustrate the impacts of the two different stochastic interest rate modeling assumptions, say the Ho-Lee model and the Vasicek model, on the optimal decision making of the insurer. It is found that the presence of mean-reverting effect in the stochastic interest rate described by the Vasicek model reduces the optimal amount of money the insurer should invest in the bond. Inspired by Bui et al. [46] and Wang et al. [47], we will study our problem under the game frameworks or more complex hybrid stochastic systems in the future.

\section{Appendix}

\section{A. The Proof of Uniformly Integrability}

Proof of Lemma 1. Note that the candidate optimal controls $u^{*}(t)$ in the Ho-Lee model are the same as that in the Vasicek model (see (36) and (44)). Thus, under the candidate optimal control $\tilde{\pi}^{*}(t)=\left(\pi_{1}^{*}(t), \pi_{2}^{*}(t), u^{*}(t)\right)$, we can rewrite the wealth process (13) as

$$
\begin{aligned}
\mathrm{d} X_{t}^{*}= & X_{t}^{*}\left\{\left[r(t)+\pi_{1}^{*}(t) \eta(t) \sigma_{1}(t)+\pi_{2}^{*}(t) \sigma_{2}(t) \lambda(t)\right.\right. \\
& \left.-I(t)+\sigma_{0}^{2}(t)+\frac{c^{2}(t)}{\sigma_{3}^{2}(t)} \frac{1}{1-p}\right] \mathrm{d} t+\frac{c(t)}{\sigma_{3}(t)} \frac{1}{1-p} \mathrm{~d} W_{3}(t) \\
& +\sigma_{1}(t) \pi_{1}^{*}(t) \mathrm{d} W_{1}(t)+\sigma_{2}(t) \pi_{2}^{*}(t) \mathrm{d} W_{2}(t) \\
& \left.-\sigma_{0}(t) \mathrm{d} W_{0}(t)\right\} .
\end{aligned}
$$

From the standard stochastic differential equation theory, we have the following explicit expression of the wealth process:

$$
\begin{aligned}
X_{t}^{*}= & D_{1}(t) \cdot \exp \left\{\int_{0}^{t} r(s) \mathrm{d} s-\int_{0}^{t} I(s) \mathrm{d} s\right. \\
& +\frac{1}{1-p} \int_{0}^{t} \frac{c(s)}{\sigma_{3}(s)} \mathrm{d} W_{3}(s)+\int_{0}^{t} \sigma_{1}(s) \pi_{1}^{*}(s) \mathrm{d} W_{1}(s) \\
& \left.+\int_{0}^{t} \sigma_{2}(s) \pi_{2}^{*}(s) \mathrm{d} W_{2}(s)-\int_{0}^{t} \sigma_{0}(s) \mathrm{d} W_{0}(s)\right\},
\end{aligned}
$$

where

$$
\begin{aligned}
D_{1}(t)= & X_{0} \exp \int_{0}^{t}\left[\pi_{1}^{*}(s) \eta(s) \sigma_{1}(s)+\pi_{2}^{*}(s) \sigma_{2}(s) \lambda(s)\right. \\
& +\sigma_{0}^{2}(s)+\frac{c^{2}(s)}{(1-p) \sigma_{3}^{2}(s)}-\frac{c^{2}(s)}{2(1-p)^{2} \sigma_{3}^{2}(s)} \\
& -\frac{\sigma_{1}^{2}(s)\left(\pi_{1}^{*}(s)\right)^{2}}{2}-\frac{\sigma_{2}^{2}(s)\left(\pi_{1}^{*}(s)\right)^{2}}{2}-\frac{\sigma_{0}^{2}(s)}{2} \\
& \left.+\sigma_{1}(s) \sigma_{0}(s) \pi_{1}^{*}(s) \rho\right] \mathrm{d} s
\end{aligned}
$$

and $X_{0}$ is the initial value of the wealth process.

A.1. Ho-Lee Model. To prove the uniformly integrability of $\left\{G\left(\tau_{n}, X_{\tau_{n}}^{*}, I\left(\tau_{n}\right), r\left(\tau_{n}\right)\right)\right\}_{n \in N}$, we only need to show that for any fixed $q>1$, 


$$
\sup _{n \in N} E\left(\left|G\left(\tau_{n}, X_{\tau_{n}}^{*}, r\left(\tau_{n}\right), I\left(\tau_{n}\right)\right)\right|^{q}\right)<\infty
$$

Combining equations (35) and (A.2), we have, for every fixed $q>1$,

$$
\begin{aligned}
\left|G\left(t, X_{t}^{*}, r(t), I(t)\right)\right|^{q}= & \frac{1}{p^{q}} \exp \left\{-p q \int_{t}^{T} h(s) \mathrm{d} s+q k(t) r(t)+q z(t) I(t)\right\}\left(X_{t}^{*}\right)^{p q} \\
= & \left|D_{2}(t)\right|^{q} \exp \left\{q k(t) r(t)+q p \int_{0}^{t} r(s) \mathrm{d} s+q p \int_{0}^{t} \sigma_{1}(s) \pi_{1}^{*}(s) \mathrm{d} W_{1}(s)\right\} \\
& \times \exp \left\{q z(t) I(t)-q p \int_{0}^{t} I(s) \mathrm{d} s-q p \int_{0}^{t} \sigma_{0}(s) \mathrm{d} W_{0}(s)\right\} \\
& \times \exp \left\{\frac{q p}{1-p} \int_{0}^{t} \frac{c(s)}{\sigma_{3}(s)} \mathrm{d} W_{3}(s)\right\} \exp \left\{q p \int_{0}^{t} \sigma_{2}(s) \pi_{2}^{*}(s) \mathrm{d} W_{2}(s)\right\} .
\end{aligned}
$$

where we set

$$
D_{2}(t)=\frac{1}{p} \exp \left\{-p \int_{t}^{T} h(s) \mathrm{d} s\right\} D_{1}^{p}(t)
$$

Note that $k(t)=p(T-t), r(t) t=\int_{0}^{t} s \mathrm{~d} r(s)+\int_{0}^{t} r(s) \mathrm{d} s$, and stochastic differential equation (4) satisfied by $r(t)$, we can easily obtain

$$
\begin{aligned}
& \exp \left\{q k(t) r(t)+q p \int_{0}^{t} r(s) \mathrm{d} s+q p \int_{0}^{t} \sigma_{1}(s) \pi_{1}^{*}(s) \mathrm{d} W_{1}(s)\right\} \\
& =\exp \left\{q p T r_{0}+q p \int_{0}^{t} a(s)(T-s) \mathrm{d} s\right. \\
& \left.\quad+q p \int_{0}^{t}\left[\sigma_{1}(t) \pi_{1}^{*}(s)+b(T-s)\right] \mathrm{d} W_{1}(s)\right\} .
\end{aligned}
$$

Applying the product differential rule to $z(t) I(t)$ yields

$$
\begin{aligned}
z(t) I(t)= & z(0) I_{0}+\int_{0}^{t} I(s) z^{\prime}(s) \mathrm{d} s+\int_{0}^{t} z(s) \mathrm{d} I(s) \\
= & z(0) I_{0}+\int_{0}^{t} I(s) z^{\prime}(s) \mathrm{d} s+\int_{0}^{t} z(s)\{\beta(s)[\alpha(s) \\
& \left.-I(s)] \mathrm{d} s+\bar{\sigma}_{0}(s) \mathrm{d} W_{0}(s)\right\} \\
= & z(0) I_{0}+\int_{0}^{t} p I(s) \mathrm{d} s+\int_{0}^{t} z(s) \beta(s) \alpha(s) \mathrm{d} s \\
& +\int_{0}^{t} \bar{\sigma}_{0}(s) z(s) \mathrm{d} W_{0}(s)
\end{aligned}
$$

where we use equation (31) in the last equality. Therefore, we have

$$
\begin{aligned}
& \exp \left\{q z(t) I(t)-q p \int_{0}^{t} I(s) \mathrm{d} s-q p \int_{0}^{t} \sigma_{0}(s) \mathrm{d} W_{0}(s)\right\} \\
& =\exp \left\{q z(0) I_{0}+q \int_{0}^{t} \beta(s) \alpha(s) z(s) \mathrm{d} s\right. \\
& \left.\quad+q \int_{0}^{t} \bar{\sigma}_{0}(s) z(s) \mathrm{d} W_{0}(s)-q p \int_{0}^{t} \sigma_{0}(s) \mathrm{d} W_{0}(s)\right\}
\end{aligned}
$$

Substituting (A.8) and (A.9) into (A.5) leads to $\left|G\left(t, X_{t}^{*}, r(t), I(t)\right)\right|^{q}$

$$
\begin{aligned}
= & \left|D_{2}(t)\right|^{q} \exp \left\{q p T r_{0}+q z(0) I_{0}+q p \int_{0}^{t} a(s)(T-s) \mathrm{d} s\right. \\
& \left.+q \int_{0}^{t} \beta(s) \alpha(s) z(s) \mathrm{d} s\right\} \\
& \cdot \exp \left\{\frac{q p}{1-p} \int_{0}^{t} \frac{c(s)}{\sigma_{3}(s)} \mathrm{d} W_{3}(s)+q p \int_{0}^{t}\left[\sigma_{1}(s) \pi_{1}^{*}(s)\right.\right. \\
& +b(T-s)] d W_{1}(s)+q p \int_{0}^{t} \sigma_{2}(s) \pi_{2}^{*}(s) \mathrm{d} W_{2}(s) \\
& \left.+q \int_{0}^{t}\left[\bar{\sigma}_{0}(s) z(s)-p \sigma_{0}(s)\right] \mathrm{d} W_{0}(s)\right\} .
\end{aligned}
$$


Let

$$
\begin{aligned}
& M(t)= \exp \left\{-\frac{1}{2} \frac{q^{2} p^{2}}{(1-p)^{2}} \int_{0}^{t} \frac{c^{2}(s)}{\sigma_{3}^{2}(s)} \mathrm{d} s-\frac{1}{2} q^{2} p^{2} \int_{0}^{t}\left[\sigma_{1}(s) \pi_{1}^{*}(s)+b(T-s)\right]^{2} \mathrm{~d} s\right. \\
&-\frac{1}{2} q^{2} p^{2} \int_{0}^{t} \sigma_{2}^{2}(s)\left(\pi_{2}^{*}(s)\right)^{2} \mathrm{~d} s-\frac{1}{2} q^{2} \int_{0}^{t}\left[\bar{\sigma}_{0}(s) z(s)-p \sigma_{0}(s)\right]^{2} \mathrm{~d} s \\
&\left.-q^{2} p \rho \int_{0}^{t}\left[\sigma_{1}(s) \pi_{1}^{*}(s)+b(T-s)\right]\left[\bar{\sigma}_{0}(s) z(s)-p \sigma_{0}(s)\right] \mathrm{d} s\right\} \\
& \cdot \exp \left\{\frac{q p}{1-p} \int_{0}^{t} \frac{c(s)}{\sigma_{3}(s)} \mathrm{d} W_{3}(s)+q p \int_{0}^{t}\left[\sigma_{1}(s) \pi_{1}^{*}(s)+b(T-s)\right] \mathrm{d} W_{1}(s)\right. \\
&\left.+q p \int_{0}^{t} \sigma_{2}(s) \pi_{2}^{*}(s) \mathrm{d} W_{2}(s)+q \int_{0}^{t}\left[\bar{\sigma}_{0}(s) z(s)-p \sigma_{0}(s)\right] \mathrm{d} W_{0}(s)\right\}, \\
& D_{3}(t)=\left|D_{2}(t)\right|^{q} \exp \left\{q p T r_{0}+q z(0) I_{0}+q p \int_{0}^{t} a(s)(T-s) \mathrm{d} s+q \int_{0}^{t} \beta(s) \alpha(s) z(s) \mathrm{d} s\right\} \\
& \cdot \exp \left\{\frac{1}{2} \frac{q^{2} p^{2}}{(1-p)^{2}} \int_{0}^{t} \frac{c^{2}(s)}{\sigma_{3}^{2}(s)} \mathrm{d} s+\frac{1}{2} q^{2} p^{2} \int_{0}^{t}\left[\sigma_{1}(s) \pi_{1}^{*}(s)+b(T-s)\right]^{2} \mathrm{~d} s\right. \\
&+\frac{1}{2} q^{2} p^{2} \int_{0}^{t} \sigma_{2}^{2}(s)\left(\pi_{2}^{*}(s)\right)^{2} \mathrm{~d} s+\frac{1}{2} q^{2} \int_{0}^{t}\left[\bar{\sigma}_{0}(s) z(s)-p \sigma_{0}(s)\right]^{2} \mathrm{~d} s \\
&\left.+q^{2} p \rho \int_{0}^{t}\left[\sigma_{1}(s) \pi_{1}^{*}(s)+b(T-s)\right]\left[\bar{\sigma}_{0}(s) z(s)-p \sigma_{0}(s)\right] \mathrm{d} s\right\} . \\
& \text { sup } E\left(\left|G\left(\tau_{n}, X_{\tau_{n}}, r\left(\tau_{n}\right), I\left(\tau_{n}\right)\right)\right|^{q}\right)<\infty .
\end{aligned}
$$

It is easy to see that $\{M(t)\}_{t \geq 0}$ is a martingale and

$$
\left|G\left(t, X_{t}^{*}, r(t), I(t)\right)\right|^{q}=D_{3}(t) M(t) .
$$

Thus, by the optional stopping time theorem and the fact that $D_{3}(t)$ is deterministic and continuous on the interval $[0, T]$, we obtain that for all stopping times $\tau_{n}$ with $0 \leq \tau_{n} \leq T$

$$
\begin{gathered}
E\left(\left|G\left(\tau_{n}, X_{\tau_{n}}^{*}, r\left(\tau_{n}\right), I\left(\tau_{n}\right)\right)\right|^{q}\right)=E\left[D_{3}\left(\tau_{n}\right) \cdot M\left(\tau_{n}\right)\right] \\
\quad \leq \sup _{t \in[0, T]} D_{3}(t) \cdot E\left[M\left(\tau_{n}\right)\right] \leq \sup _{t \in[0, T]} D_{3}(t)<\infty .
\end{gathered}
$$

Therefore, uniformly integrable property follows up.

A.2. Vasicek Model. For the Vasicek model, we also need to prove that for any fixed $q>1$,

$$
\sup _{n \in N} E\left(\left|G\left(\tau_{n}, X_{\tau_{n}}^{*}, r\left(\tau_{n}\right), I\left(\tau_{n}\right)\right)\right|^{q}\right)<\infty .
$$

Combining equations (43) and (A.2), we have for every fixed $q>1$,

Note that $\sup _{t \in[0, T]} D_{3}(t)<\infty$ is independent of $n$. Thus, taking supremum over $n \in N$ on both sides of the above equation yields

$$
\begin{aligned}
\left|G\left(t, X_{t}^{*}, r_{t}, I_{t}\right)\right|^{q}= & \frac{1}{p^{q}} \exp \left\{-p q \int_{t}^{T} \tilde{h}(s) \mathrm{d} s+q \tilde{k}(t) r(t)+q \widetilde{z}(t) I(t)\right\}\left(X_{t}^{*}\right)^{p q} \\
= & \left|\widetilde{D}_{2}(t)\right|^{q} \exp \left\{q \widetilde{k}(t) r(t)+q p \int_{0}^{t} r(s) \mathrm{d} s+q p \int_{0}^{t} \sigma_{1}(s) \pi_{1}^{*}(s) \mathrm{d} W_{1}(s)\right\} \\
& \times \exp \left\{q \widetilde{z}(t) I(t)-q p \int_{0}^{t} I(s) \mathrm{d} s-q p \int_{0}^{t} \sigma_{0}(s) \mathrm{d} W_{0}(s)\right\} \\
& \times \exp \left\{\frac{q p}{1-p} \int_{0}^{t} \frac{c(s)}{\sigma_{3}(s)} \mathrm{d} W_{3}(s)\right\} \exp \left\{q p \int_{0}^{t} \sigma_{2}(s) \pi_{2}^{*}(s) \mathrm{d} W_{2}(s)\right\}
\end{aligned}
$$


where we let

$$
\widetilde{D}_{2}(t)=\frac{1}{p} \exp \left\{-p \int_{t}^{T} \tilde{h}(s) \mathrm{d} s\right\} D_{1}^{p}(t) .
$$

Note that $\widetilde{z}(t)$ in the Vasicek model is equal to $z(t)$ in the Ho-Lee model and so the difference between the expression of $\left|G\left(t, X_{t}^{*}, r_{t}, I_{t}\right)\right|^{q}$ in the Vasicek model and Ho-Lee model is the first part of the expression of $\left|G\left(t, X_{t}^{*}, r_{t}, I_{t}\right)\right|^{q}$.

Since in the Vasicek model, $r(t)$ is described by

$$
\mathrm{d} r(t)=[\theta(t)-\widehat{b} r(t)+b \xi(t)] \mathrm{d} t+b \mathrm{~d} W_{1}(t),
$$

and $\widetilde{k}(t)=(p / \widehat{b})\left[1-e^{\widehat{b}(t-T)}\right]$; after some calculations, we can easily get

$$
\begin{aligned}
& \exp \left\{q \widetilde{k}(t) r(t)+q p \int_{0}^{t} r(s) \mathrm{d} s+q p \int_{0}^{t} \sigma_{1}(s) \pi_{1}^{*}(s) \mathrm{d} W_{1}(s)\right\} \\
= & \exp \left\{-\frac{q p}{\widehat{b}} e^{-\widehat{b} T} r_{0}-\frac{q p}{\widehat{b}} \int_{0}^{t} e^{\widehat{b}(s-T)}[\theta(s)+b \xi(s)] \mathrm{d} s+\frac{q p}{\widehat{b}} r_{0}\right. \\
& \left.+\frac{q p}{\widehat{b}} \int_{0}^{t}[\theta(s)+b \xi(s)] \mathrm{d} s\right\} \\
& \times \exp \left\{\int_{0}^{t}\left[\frac{b}{\widehat{b}}+q p \sigma_{1}(s) \pi_{1}^{*}(s)-\frac{q p}{\widehat{b}} e^{\widehat{b}(s-T)} b\right] \mathrm{d} W_{1}(s)\right\} .
\end{aligned}
$$

Now, following the same method used in the Ho-Lee model, we can finally find a deterministic and continuous function $\widetilde{D}_{3}(t)$ and a martingale $\widetilde{M}(t)$ such that

$$
\left|G\left(t, X_{t}^{*}, r(t), I(t)\right)\right|^{q}=\widetilde{D}_{3}(t) \tilde{M}(t) .
$$

Similar to the method used in the Ho-Lee model, we can obtain the uniformly integrable property of $\left\{G\left(\tau_{n}, X_{\tau_{n}}^{*}, r\left(\tau_{n}\right), I\left(\tau_{n}\right)\right)\right\}_{n \in N^{*}}$

Thus, we complete our proof.

\section{Data Availability}

The data used to support the findings of this study are included within the article.

\section{Conflicts of Interest}

The authors declare that they have no conflicts of interest.

\section{Authors' Contributions}

All authors contributed equally to the writing of this paper. All authors read and approved the final version.

\section{Acknowledgments}

This work was supported by the National Natural Science Foundation of China (grant nos. 11771079 and 11371020).

\section{References}

[1] H. Markowitz, "Portfolio selection," The Journal of Finance, vol. 7, no. 1, pp. 77-91, 1952.
[2] R. C. Merton, "Lifetime portfolio selection under uncertainty: the continuous-time case," The Review of Economics and Statistics, vol. 51, no. 3, pp. 247-257, 1969.

[3] R. C. Merton, "Optimum consumption and portfolio rules in a continuous-time model," Journal of Economic Theory, vol. 3, no. 4, pp. 373-413, 1971.

[4] T. K. Siu, "Long-term strategic asset allocation with inflation risk and regime switching," Quantitative Finance, vol. 11, no. 10, pp. 1565-1580, 2011.

[5] C. Robert, Continuous-Time Finance, Blackwell, Oxford, UK, 1990.

[6] K. Ralf, Optimal Portfolios: Stochastic Models for Optimal Investment and Risk Management in Continuous Time, World Scientific, Singapore, 1997.

[7] I. Karatzas and E. Steven, Shreve. Methods of Mathematical Finance, Springer, New York, NY, USA, 1998.

[8] R. J. Elliott and P. E. Kopp, Mathematics of Financial Markets, Springer Finance, New York, NY, USA, 2005.

[9] M. H. A. Davis and A. R. Norman, "Portfolio selection with transaction costs," Mathematics of Operations Research, vol. 15, no. 4, pp. 676-713, 1990.

[10] J. F. Cocco, F. J. Gomes, and P. J. Maenhout, "Consumption and portfolio choice over the life cycle," Review of Financial Studies, vol. 18, no. 2, pp. 491-533, 2005.

[11] H. Kraft, "Optimal portfolios and Heston's stochastic volatility model: an explicit solution for power utility," Quantitative Finance, vol. 5, no. 3, pp. 303-313, 2005.

[12] J. Y. Campbell and L. M. Viceira, Strategic Asset Allocation: Portfolio Choice for Long-Term Investors. Clarendon Lectures in Economics, Oxford University Press, Oxford, UK, 2002.

[13] A. D. Wilkie, "A stochastic investment model for actuarial use," Transactions of the Faculty of Actuaries, vol. 39, pp. 341-403, 1984.

[14] N. D. Pearson and T.-S. Sun, "Exploiting the conditional density in estimating the term structure: an application to the Cox, Ingersoll, and Ross model," The Journal of Finance, vol. 49, no. 4, pp. 1279-1304, 1994.

[15] C. Munk, C. Sørensen, and T. Nygaard Vinther, "Dynamic asset allocation under mean-reverting returns, stochastic interest rates, and inflation uncertainty," International Review of Economics \& Finance, vol. 13, no. 2, pp. 141-166, 2004.

[16] M. J. Brennan and Y. Xia, "Dynamic asset allocation under inflation," The Journal of Finance, vol. 57, no. 3, pp. 1201-1238, 2002.

[17] R. Korn, T. K. Siu, and A. Zhang, "Asset allocation for a DC pension fund under regime switching environment," European Actuarial Journal, vol. 1, no. S2, pp. 361-377, 2011.

[18] T. K. Siu, “A BSDE approach to risk-based asset allocation of pension funds with regime switching," Annals of Operations Research, vol. 2012, no. 1, pp. 449-473, 2012.

[19] R. Korn and H. Kraft, "A stochastic control approach to portfolio problems with stochastic interest rates," SIAM Journal on Control and Optimization, vol. 40, no. 4, pp. 1250-1269, 2002.

[20] J. Li and R. Wu, "Optimal investment problem with stochastic interest rate and stochastic volatility: maximizing a power utility," Applied Stochastic Models in Business and Industry, vol. 25, no. 3, pp. 407-420, 2009.

[21] H. Kraft, "Optimal portfolios with stochastic short rate: pitfalls when the short rate is non-gaussian or the market price of risk is unbounded," International Journal of Theoretical and Applied Finance, vol. 12, no. 6, pp. 767-796, 2009.

[22] Y. Shen and T. K. Siu, "Asset allocation under stochastic interest rate with regime switching," Economic Modelling, vol. 29, no. 4, pp. 1126-1136, 2012. 
[23] Z. Wang, J. Xia, and L. Zhang, "Optimal investment for an insurer: the martingale approach," Insurance: Mathematics and Economics, vol. 40, no. 2, pp. 322-334, 2007.

[24] X. Zeng, "A stochastic differential reinsurance game," Journal of Applied Probability, vol. 47, no. 2, pp. 335-349, 2010.

[25] S. Luo, M. Taksar, and A. Tsoi, "On reinsurance and investment for large insurance portfolios," Insurance: Mathematics and Economics, vol. 42, no. 1, pp. 434-444, 2008.

[26] R. J. Elliott and T. K. Siu, "A BSDE approach to a risk-based optimal investment of an insurer," Automatica, vol. 47, no. 2, pp. 253-261, 2011.

[27] Z. Liang, K. C. Yuen, and K. C. Cheung, "Optimal reinsurance-investment problem in a constant elasticity of variance stock market for jump-diffusion risk model," Applied Stochastic Models in Business and Industry, vol. 28, no. 6, pp. 585-597, 2012.

[28] R. J. Elliott and T. K. Siu, "An HMM approach for optimal investment of an insurer," International Journal of Robust and Nonlinear Control, vol. 22, no. 7, pp. 778-807, 2011.

[29] T. K. Siu, "A BSDE approach to optimal investment of an insurer with hidden regime switching," Stochastic Analysis and Applications, vol. 31, no. 1, pp. 1-18, 2013.

[30] L. Bai and H. Zhang, "Dynamic mean-variance problem with constrained risk control for the insurers," Mathematical Methods of Operations Research, vol. 68, no. 1, pp. 181-205, 2008.

[31] X. Zhang and T. K. Siu, "Optimal investment and reinsurance of an insurer with model uncertainty," Insurance: Mathematics and Economics, vol. 45, no. 1, pp. 81-88, 2009.

[32] X. Zhang and T. K. Siu, "On optimal proportional reinsurance and investment in a Markovian regime-switching economy," Acta Mathematica Sinica, English Series, vol. 28, no. 1, pp. 67-82, 2012.

[33] X. Zhang, R. J. Elliott, and T. K. Siu, “A Bayesian approach for optimal reinsurance and investment in a diffusion model," Journal of Engineering Mathematics, vol. 76, no. 1, pp. 195206, 2012.

[34] J. Liu, K.-F. Cedric Yiu, T. K. Siu, and W.-K. Ching, "Optimal investment-reinsurance with dynamic risk constraint and regime switching," Scandinavian Actuarial Journal, vol. 2013, no. 4, pp. 263-285, 2013.

[35] T. B. Douglas, "Consumption, production, inflation and interest rates," Journal of Financial Economics, vol. 16, no. 1, pp. 3-39, 1986.

[36] G. George, "Identifying the dynamics of real interest rates and inflation: Evidence using survey data," Review of Financial Studies, vol. 4, no. 1, pp. 53-86, 1991.

[37] E. F. Fama and M. R. Gibbons, "Inflation, real returns and capital investment," Journal of Monetary Economics, vol. 9, no. 3, pp. 297-323, 1982.

[38] H. Yao, Z. Yang, and P. Chen, "Markowitz's mean-variance defined contribution pension fund management under inflation: a continuous-time model," Insurance: Mathematics and Economics, vol. 53, no. 3, pp. 851-863, 2013.

[39] G. Jan, Aspects of Risk Theory, Springer, New York, NY, USA, 1991.

[40] Y. Zeng and Z. Li, "Optimal time-consistent investment and reinsurance policies for mean-variance insurers," Insurance: Mathematics and Economics, vol. 49, no. 1, pp. 145-154, 2011.

[41] B. Højgaard and M. Taksar, "Optimal proportional reinsurance policies for diffusion models," Scandinavian Actuarial Journal, vol. 1998, no. 2, pp. 166-180, 1998.
[42] I. T. Michael and C. Markussen, "Optimal dynamic reinsurance policies for large insurance portfolios," Finance and Stochastics, vol. 7, no. 1, pp. 97-121, 2003.

[43] I. Karatzas and S. E. Shreve, Brownian Motion and Stochastic Calculus, Springer, New York, NY, USA, 1991.

[44] F. Wendell, Deterministic and Stochastic Optimal Control, R. W. Rishel and F. Wendell, Eds., Springer, New York, NY, USA, 1975.

[45] H. F. Wendell, "Controlled markov processes and viscosity solutions," in Stochastic Modelling and Applied Probability, F. Wendell and H. M. Soner, Eds., Springer, New York, NY, USA, 2nd edition, 2006.

[46] T. Bui, X. Cheng, Z. Jin, and G. Yin, "Approximation of a class of non-zero-sum investment and reinsurance games for regime-switching jump-diffusion models," Nonlinear Analysis: Hybrid Systems, vol. 32, pp. 276-293, 2019.

[47] N. Wang, N. Zhang, Z. Jin, and L. Qian, "Robust non-zerosum investment and reinsurance game with default risk," Insurance: Mathematics and Economics, vol. 84, pp. 115-132, 2019. 


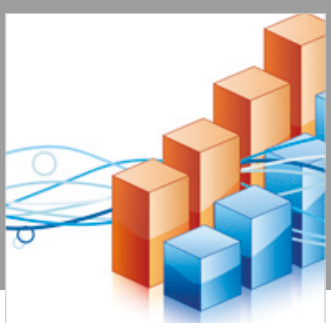

Advances in

Operations Research

\section{-n-m}
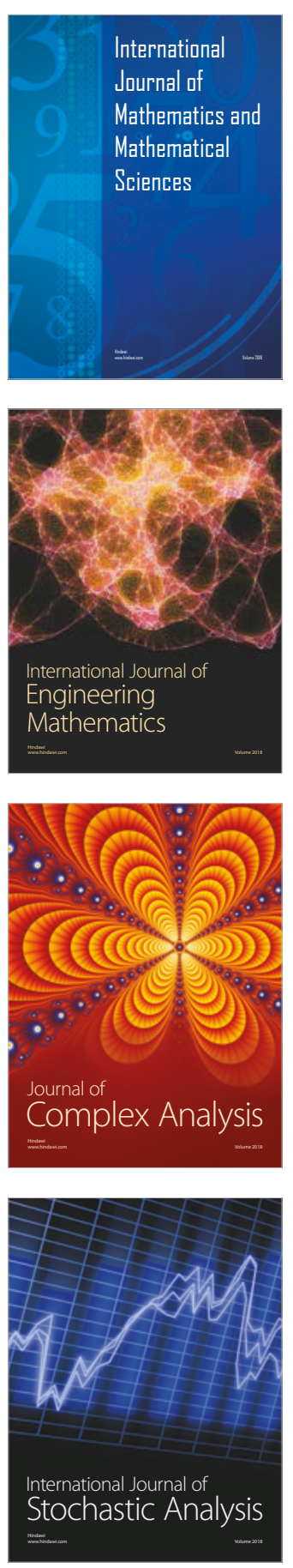
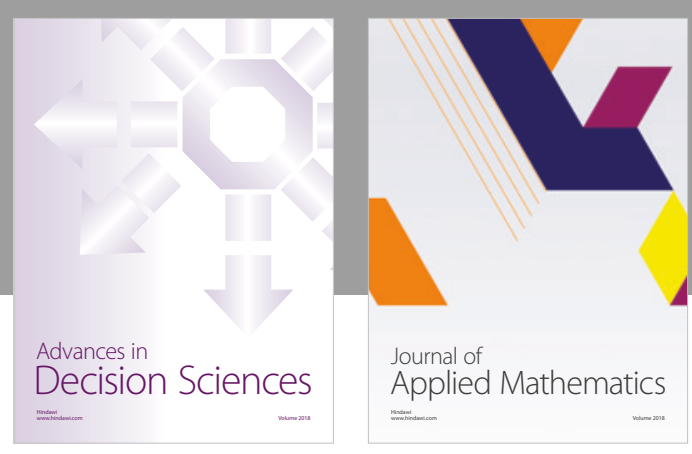

Journal of

Applied Mathematics
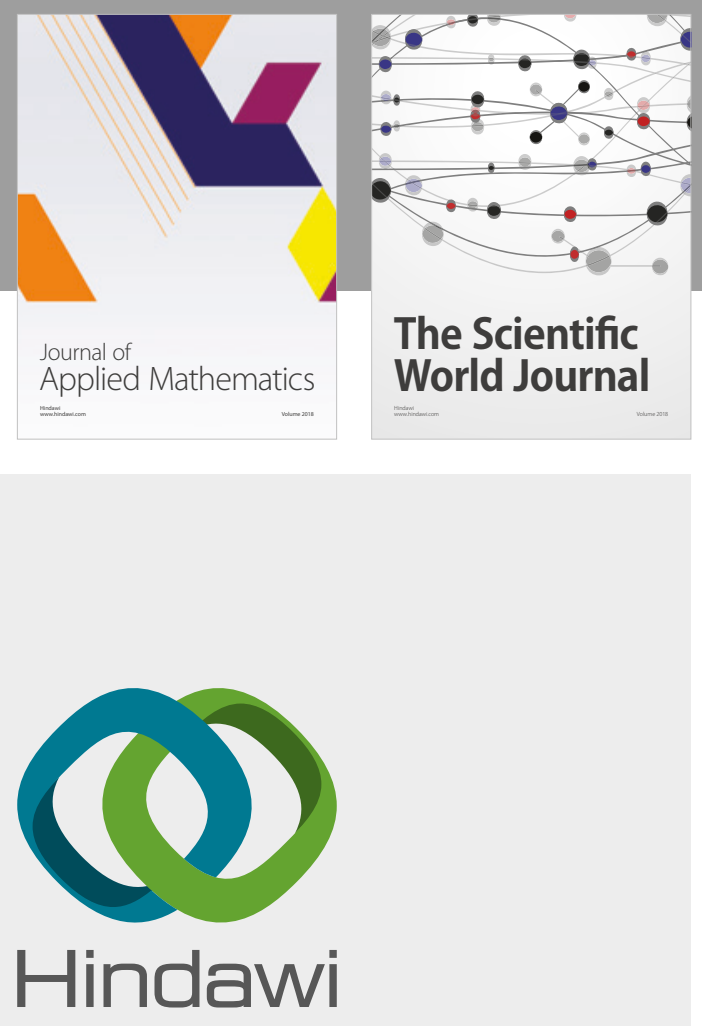

Submit your manuscripts at

www.hindawi.com

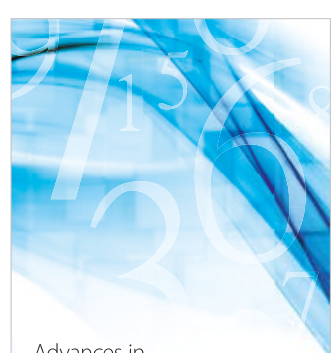

Advances in
Numerical Analysis
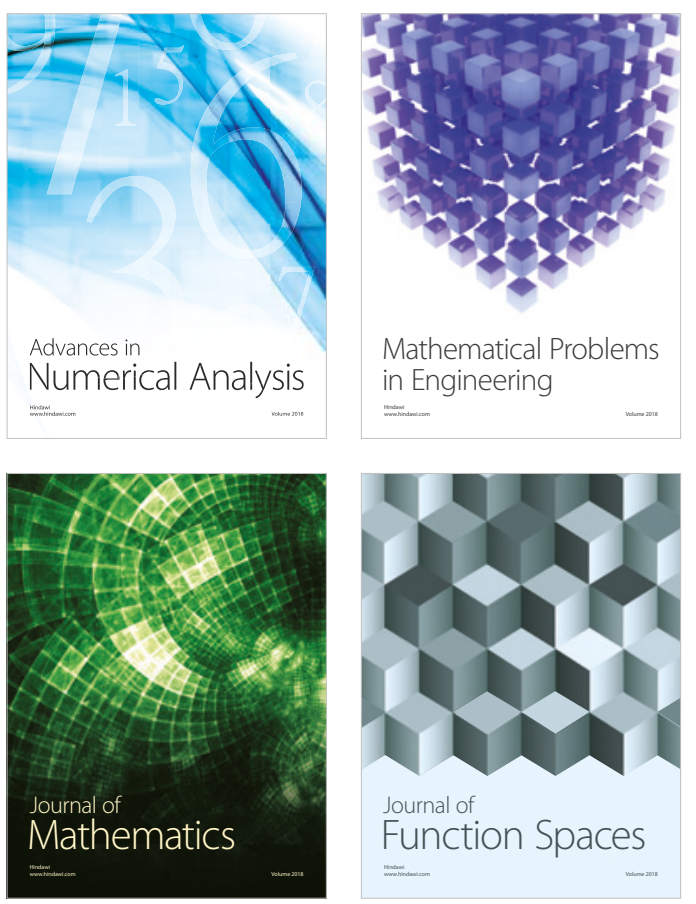

Mathematical Problems in Engineering

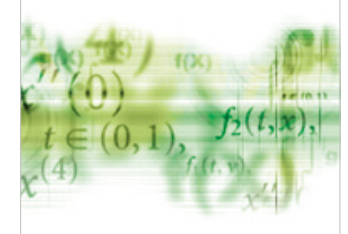

International Journal of

Differential Equations

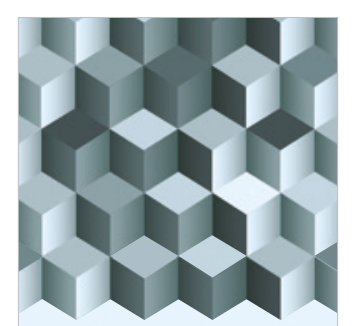

Journal of

Function Spaces

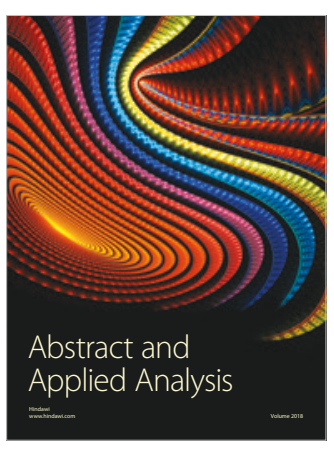

The Scientific

World Journal

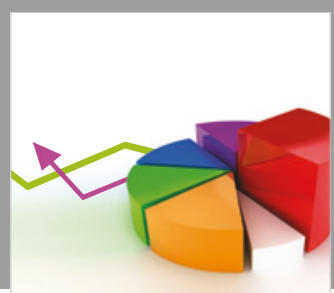

Journal of

Probability and Statistics
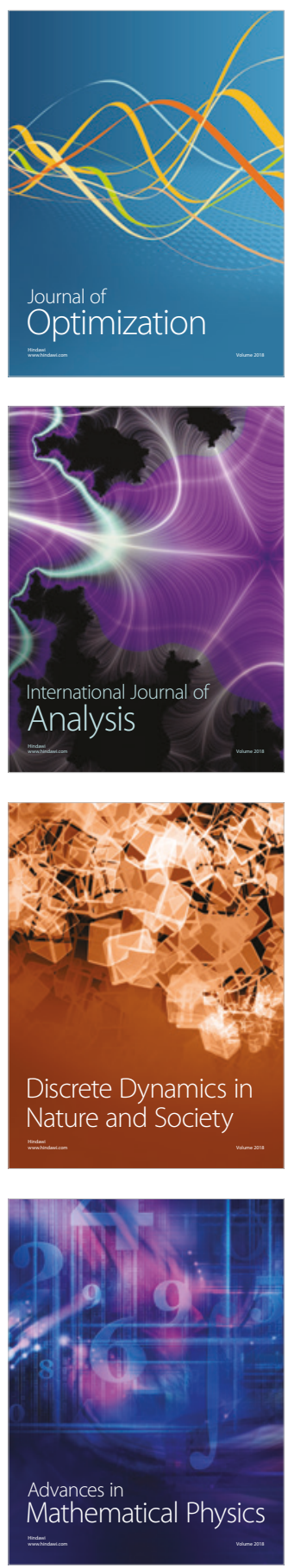